\title{
Design of Truss-like Cellular Structures Using Relative Density Mapping Method
}

\author{
Mahmoud Alzahrani ${ }^{1 *}$, Seung-Kyum Choi ${ }^{2 *}$ and David W. Rosen ${ }^{3 *}$ \\ * G. W. Woodruff School of Mechanical Engineering, Georgia Institute of Technology, \\ Atlanta, GA, 30332, USA
}

\begin{abstract}
-
Truss-like cellular structures have great potential to be applied in light-weight design applications. However, determining the appropriate designs for these truss-like cellular structures can be a challenging task due to their geometric complexities and prohibitive computational costs in the design process. In this research, a new design method is proposed which can drastically reduce computational costs and design parameters, while maintaining the performance of the targeted outcome. Furthermore, the proposed method facilitates cellular structures designs that can handle multiple loading conditions. The proposed method utilizes the relative density information obtained from a solid topology optimization to automatically determine the diameter of each individual strut in the structure, which collectively represent the set of design variables. This allows the method to produce lattice structures that can perform reliably under multiple loading conditions and also reduce the computational cost associated with the design of these structures. The efficacy of the developed method is compared to existing methods including the size matching and scaling method that combines solid-body analysis and a predefined unit-cell library.
\end{abstract}

Key words: Cellular structures, Lattice structures, Topology optimization, Additive manufacturing, Relative density mapping.

\section{Nomenclature}

$\begin{array}{ll}A r & \text { Relative area of struts } \\ \text { Ar } & \text { Relative area of struts vector } \\ A r_{l} & \text { Lower limit of relative area } \\ \text { BCN } & \text { Boundary condition nodes vector } \\ D & \text { Strut diameter } \\ \text { D } & \text { Struts' diameter vector } \\ \text { EC } & \text { Centroidal coordinates of elements } \\ L & \text { Length of strut } \\ \text { LDN } & \text { Loaded nodes vector } \\ n & \text { Number of elements in topology optimization } \\ N & \text { Number of struts in lattice structure } \\ & \\ 1 & \\ 2 & \\ \text { Associate Professor, 813 Ferst Drive N. W., Atlanta, GA 30332 }\end{array}$




$\begin{array}{ll}\mathbf{r} & \text { Distance array } \\ r_{i j} & \text { Distance from element } i \text { to strut } j \\ R R & \text { Rejection ratio } \\ S f & \text { Scaling factor } \\ V & \text { Lattice structure volume } \\ V_{0} & \text { Design volumetric constraint } \\ w & \text { Weight function } \\ w_{d} & \text { Displacement weight variable } \\ w_{v} & \text { Volume weight variable } \\ \delta & \text { Displacement } \\ \rho & \text { Element's relative density in topology optimization } \\ \rho & \text { Relative density vector } \\ \rho_{s} & \text { Density of cellular structure's solid counterpart } \\ \rho_{v} & \text { Volumetric density of cellular structure } \\ \rho^{*} & \text { Density of cellular structure }\end{array}$

\section{Introduction and Background}

Recent advancements in additive manufacturing (AM) have allowed designers to achieve designs that have complex geometries. In particular, cellular structures have benefited from the advances of AM. These types of structures are useful for achieving light-weight but strong structural systems, since they can be designed for very specific applications by adding material only where needed. Previously, a size matching and scaling (SMS) method [1], which incorporates solid-body finite element analysis and a unit cell library, was improved to address the drawbacks of its lack of a systematic approach in determining the appropriate diameters for the structure's struts. In this study, a novel method is developed by utilizing material density information, which is a by-product of continuum topology optimization, to identify the layout of a given structural system. The following sections will briefly describe the background of the approaches and details of the developed method. The advantages from the developed method are fully demonstrated in Section 3. The following sections will briefly describe the background of the approaches and details of the developed method.

\subsection{Additive Manufacturing}

AM processes, also known as direct manufacturing, possess unique characteristics that are not shared by conventional manufacturing processes. They are a collection of processes that fabricate 3-D objects from 2-D cross-sectional layers [2]. It was first introduced as a rapid prototyping method (RP) in the 1980's to produce rough physical prototypes of products [3]. The process may take a few hours to a few days to produce the required part depending on its size and precision. AM processes enable parts to be manufactured with high geometric complexity [4]. Other advantages of AM processes are that it has a predictable time for the fabrication of the model even when there are modifications in the design, the number of processes and resources required is significantly less than in conventional manufacturing, and it can be used to simplify multi-stage processes [5]. These advantages show great potential for utilizing AM processes in manufacturing cellular structures. A critical challenge in utilizing 
$\mathrm{AM}$, however, is the determination of appropriate designs of the cellular structures. Therefore, the focus of the presented research is on the development of new design methods for cellular structures.

\subsection{Cellular Structures}

Cellular structures, such as foams, honeycombs and lattices, are structures with interconnected struts or plates. They can contain material only where it is needed for particular applications. They are structured as repeated cells, which enables a high degree of optimization that gives designers the ability to control material distribution. The key advantages of cellular structures are their high strength relative to their low mass, good energy absorption and high thermal and acoustic insulation compared to their solid counter-parts [6]. They can be a 2-D array of polygons, such as honeycombs, 3-D closed cell foams or 3-D open cell lattice structures [7]. Lattice structures have received a considerable amount of attention over foams for their ability to produce light-weight and strong materials when compared to foams [8]. They have recently gained attention as a candidate core material for composite structures $[9,10]$. The strength of cellular structures is governed by their volumetric density $\rho_{v}$.

$$
\rho_{v}=\rho * / \rho_{s}
$$

where $\rho^{*}$ is the density of the cellular structure, and $\rho_{s}$ is the density of the solid counter-part. The strength of foams is governed by the bending of face walls that separate the cells and scales as $\rho_{v}{ }^{1.5}$. As for lattice structures, their strength is governed by the deformation of cells through stretching of the interconnecting struts, and scales as $\rho_{v}$. Thus, for cellular structures with $\rho_{v}=0.1$, lattice structures are three times stronger than foams $[6,11]$.

Various studies have been conducted to investigate lattice structures. Wallach and Gibson [12] analyzed a sheet of lattice structures with a thickness of one unit-cell. The model assumed that the struts of the lattice structure were connected with pin joints to act like truss structures. This assumes that struts only carry axial loads. This assumption is justified since the stretching forces on the struts dominate the bending forces. The sheet was subject to tensile tests in all three directions, and the results were compared to experimental results with errors of $3 \%$ to $27 \%$ depending on the direction of loading. Chiras et al. [13] extended the analysis to investigate the behavior of lattice structures under bending and shear loadings using tetragonal unit-cells. Deshpande et al. [11] conducted extensive investigation of octet unit-cell strength. Analytical models and numerical FE models were created and compared with experimental tests, which showed good agreement between the predicated properties and tests. The analytical model assumed pin jointed connections while the numerical FE model utilizes beam elements. Wang et al. [14] extended the analytical model of the octet unit-cell to take into account bending, non-linearity and buckling.

For designing lattice structures, several existing methods, including Michell's approach [15], have been considered. However, its applicability was limited since it is restricted to 2-D scenarios. The ground structure optimization approach was also used to determine the size of each individual strut in the lattice structure [16, 17]. The process utilizes optimization algorithms, such as active set [18] and Levenberg-Marquardt (LM) [19]. In general, this process is known to be computationally expensive; the complexity of the problem increases exponentially as the number of design variables is increased. These limitations motivated the need for methods that would reduce the cost in designing the lattice structures. A method called size matching and scaling (SMS) was developed and was able to reduce the 
optimization process to a 2-variable optimization [1]. This method utilizes solid-body finite elements analysis (FEA) to determine the size of individual struts. One of the draw backs of the method is that the generated lattice structure is limited to only one load case, which is the one it was designed for. The following section summarizes the basis of the SMS method.

\subsection{The SMS Method}

The SMS method [20] combines solid-body analysis and a pre-defined library of unit cells for specific loading conditions to generate the lattice structure topology. The method matches optimized unit-cells to the stress information on the nodes from the finite element method (FEM) that are bounding the region of the unit cell. It was limited to simple axis-aligned structures, and a $28 \%$ ratio between the maximum and minimum diameter is assumed to generate the lattice structure. Chang et al. [21] improved the limitations of SMS by including an updated unit-cell library of different configurations. Also, the $28 \%$ assumption was replaced by utilizing a two-variable optimization process in determining the appropriate structure's minimum and maximum diameter. This optimization process used two algorithms; namely, Levenberg-Marquardt (LM) and Active-set methods. The LM method is used for solving non-linear least squares problems. It interpolates between the Gauss Newton method and gradient descent method [22]. Active-set is a mathematical programming algorithm that uses gradient information for optimizing constrained problems [18]. Further improvement to SMS was realized by Nguyen et al. [1] to allow the generation of lattice structures for complex shapes. Although, these efforts have improved the initial limitations of the SMS method, the design of lattice structures obtained from SMS is only applicable to support a targeted loading condition. Currently, it does not provide a way of handling multiple loading conditions. Therefore, the proposed method aims to achieve lattice structures that are capable of handling multi-loading conditions while maintaining computational efficiency. This can be achieved by utilizing the relative density information obtained from by-products of the topology optimization. Again, the proposed method will not require additional computational costs once the optimal layout result of the topology optimization is available, nor will it require special constraints on the topology optimization process. The details of the proposed method are discussed in Section 2.

\subsection{Topology Optimization}

Topology optimization, also referred to as layout optimization, seeks to find the optimal load path for a certain boundary condition, which enables finding the best material distribution in the part that fulfills certain constraints, such as compliance, displacement, etc. [23]. In the late 1980's, extensive research was conducted on topology optimization [24]. The material distribution is based on the values of the relative density, $\rho$, of each element, which ranges from $\rho=0$ (void) to $\rho=1$ (solid). This allows for the creation of voids and new holes in the part where needed, which causes topological changes in the geometry without the need to re-mesh the FE model. Also, it enables the simplification of the optimization routine [25]. Results obtained from the optimization process would still require further processing. Shape optimization is carried out after extracting the model from the optimization process. This step is taken to reduce stress concentrations and smooth the edges of the part before fabrication. Many optimization approaches are used, such as Homogenization Based Optimization (HBO), Solid Isotropic Microstructure with Penalization (SIMP) and Rational Approximation of Material Properties (RAMP) [24, 26-28]. Specifically, the FEM software, ABAQUS, is 
employed to conduct the topology optimization in the paper using the SIMP technique. Using the homogenization technique would be disadvantageous since it increases the number of variables per element in contrast to SIMP. Each element would be treated as a micro-structure that may be similar to a lattice unit-cell, which would require an additional homogenization step after the optimal solution is found [29]. Also, using the homogenization technique with elements with the same size of the desired unit-cells would reduce it to a traditional ground structure optimization method.

The motivation of the work in this paper is to develop a method that can be used in generating lattice structures that reliably handle multiple loading conditions and with reduced computational cost. Existing methods lack the ability to achieve both objectives reasonably. The proposed method aims to utilize the relative density information, obtained from topology optimization, in designing lattice structures. If the relative density information is already available, re-running the topology optimization process is not required, which would eliminate the computational cost associated with the optimization process.

\section{Proposed Relative Density Mapping Method (RDM)}

\subsection{Formulation of RDM}

The relative density mapping (RDM) method uses the relative density information of finite elements used in the topology optimization process. Figure 1 shows a strut of a lattice structure that is surrounded by elements with different relative density values. The key principle of RDM is that struts that are surrounded with high relative density elements will have a higher relative cross-sectional area compared to struts surrounded with low relative density elements.

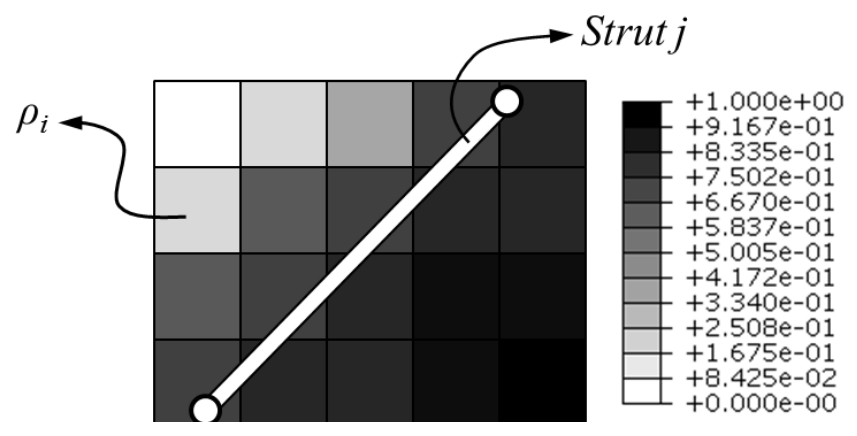

Figure 1 Relative density mapping to strut diameter

The mathematical formulation to relate the struts' relative cross-sectional area and the elements' relative density is based on the following conditions:

- Elements that are closer to the strut will influence its relative cross-sectional area more than those that are far from the strut.

- The relative cross-section value of the strut must depend on the contribution of the influence of the relative densities of all elements.

- The upper value of the strut's relative cross-sectional area is 1 .

From the first two conditions, a weighted average of the relative densities would be an appropriate formulation, where the weight function depends on the distance of the elements from the strut. 


$$
A r_{j}=\frac{\sum_{i=1}^{n} \rho_{i} w\left(r_{i j}\right)}{\sum_{i=1}^{n} w\left(r_{i j}\right)}
$$

where $A r_{j}$ is the relative cross-sectional area of strut $j, w$ is a weight function and $r_{i j}$ is the distance of element $i$ from strut $j$. An exponential function is used as a weight function where it would have a value of 1 when the distance between the strut and the element is 0 , and a decaying value as the element gets farther away from the strut. This would allow the third condition to be met. To obtain a non-dimensional form, the exponent of the weight function is divided by $10 \%$ of the strut's length, $L$. This will give elements that are within that distance higher weights.

$$
w\left(r_{i j}\right)=\exp \left(-\frac{r_{i j}}{0.1 \cdot L}\right)
$$

The centroidal coordinates of the elements were used to determine the distance of the elements from each strut. Each strut is treated as a line segment with certain length $L$ so that $r_{i j}$ is the shortest distance between the element's centroid $(E c)$ and the line segment. An illustration of this process is shown in Figure 2.

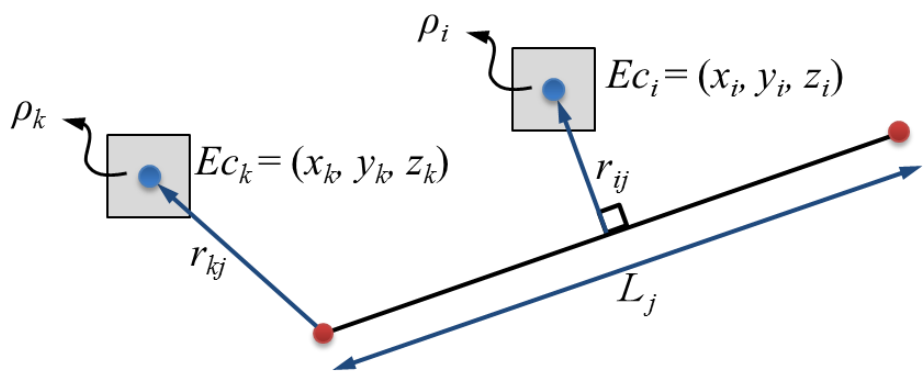

Figure 2 Distance of the centroid of element $i$ from strut $j$

\subsection{Overview of the process}

The overall process of the proposed RDM method is summarized in Figure 3. The process starts with two inputs: the information from topology optimization result, and a coarse FE mesh of the design region. The topology optimization input includes the relative density vector, $\rho$, and data from the FEM mesh, which contains the elements' nodal coordinates and connectivity data. The second input contains the nodal coordinates and connectivity data for a coarse mesh which will be used to generate a base lattice structure with $N$ struts. A crossed unit-cell type was used in this paper for the base lattice structure, which is shown in Figure 4. This unit-cell type can be replaced with other types depending upon the designer's preference. The elements' centroidal coordinates are then calculated and stored in vector $\mathbf{E} \mathbf{c}$ which is used to calculate the distance vector $\mathbf{r}_{j}$ from strut $j$. The relative cross-sectional area $A r_{j}$ is then calculated using $\rho$ and $\mathbf{r}_{\mathbf{j}}$. The process then continues until all struts' relative cross-sections are determined. Then, a screening process can be conducted which could enhance the performance of the generated structure. This screening process is optional and details are discussed in Section 2.3. 
Since the FEM analysis will use beam elements, numerical stability issues may arise from ill-conditioned stiffness matrices. Struts with extremely small relative cross-section values would cause this problem. To avoid this issue, a lower value for the relative cross-sectional area, $A r_{l}$, is set. Then, the values of the vector $\mathbf{A r}$ are scaled and adjusted such that

$$
\begin{aligned}
& \min (\mathbf{A r})=A r_{l} \\
& \max (\mathbf{A r})=1
\end{aligned}
$$

This process is done so that the ratio between any two differences between the relative areas remains the same after the adjustment. This scaling and adjustment of the values can be performed according to:

$$
A r=\underbrace{\left[\frac{1-A r_{l}}{\max (\mathbf{A r})-\min (\mathbf{A r})}\right]}_{\text {Scaling part }} \times \underbrace{[A r-\max (\mathbf{A r})]+1}_{\text {Adjustment part }}
$$

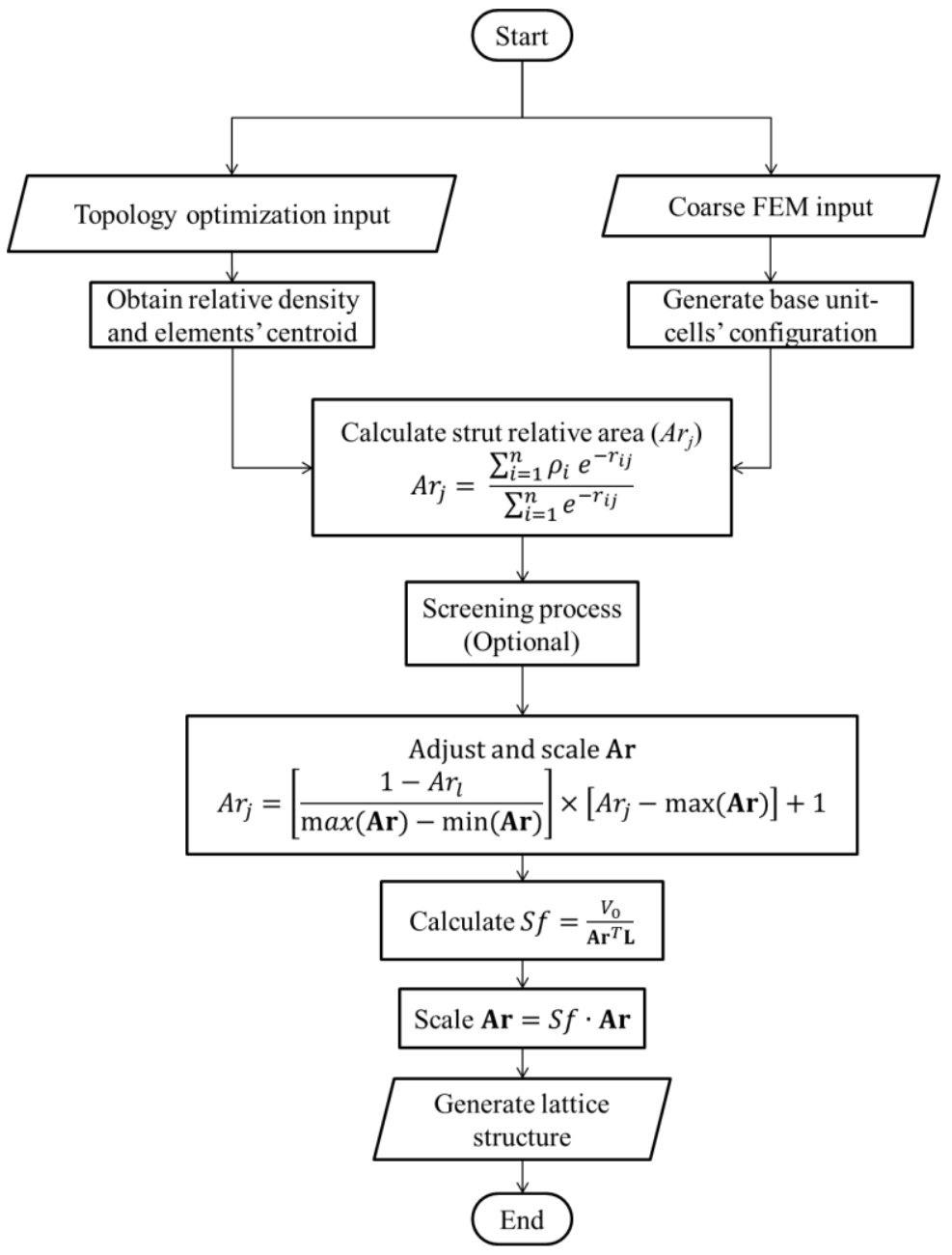

Figure 3 RDM method flowchart

The last step in the RDM method is calculating the actual cross-sectional area of each strut. A scaling factor $S f$ is calculated to determine the appropriate values for the struts' cross-sectional areas. The volume design constraint, $V_{0}$, is used to ensure that the total volume 
of the generated lattice structure meets the volume constraint. Then the values of Ar are scaled accordingly,

$$
S f=\frac{V_{0}}{\sum_{j=1}^{N} A r_{j} \times L_{j}}
$$

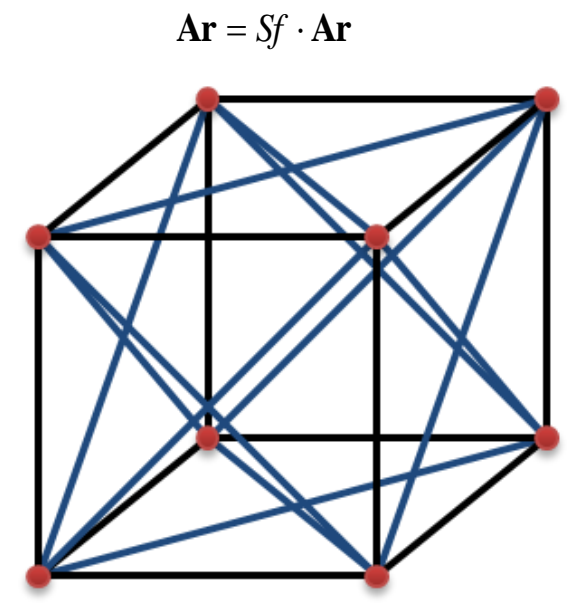

Figure 4 Crossed unit-cell structure

By utilizing Eqs. (2)-(7), the lattice structure can be generated. Before the scaling and adjustment process, the performance of the obtained design can be improved by removing nonessential struts. Thus, the additional screening process is also proposed in the next section.

\subsection{Screening Process}

This process is an optional step in the proposed RDM method. It is used to remove any nonessential struts that will not contribute to the overall performance of the structure. In general, struts that have relatively small values of $A r$ can be removed. Figure 5 shows a flow chart of the proposed screening process. The process can be initiated by identifying nodes for loading and boundary conditions. In the developed algorithm, the information is stored in vectors of LDN and BCN, respectively. Struts that contain loaded nodes are fixed so that they are not affected by the screening process, while struts with both nodes fixed are removed. A rejection ratio $R R$ is then set such that any strut with $A r<R R$ is removed. It is important to note that large values of $R R$ should be avoided to prevent the removal of critical struts.

After this initial screening process, there still exists unwanted struts which have values of $A r$ $>R R$. These struts can be removed by conducting an iterative process as shown in Figure 5 . First, free nodes which are contained in only one strut can be identified. Then, any nodes connecting two struts, which share a common third strut, can be removed. This iterative process continues until no nodes remain that fit these two criteria. The structure is assumed to be pin jointed in the proposed algorithm. This assumption will not affect the performance of the structure in the case of a node connecting two struts. Although it would contribute to the structure's stiffness when it is assumed to be rigid, the corresponding contribution would be 


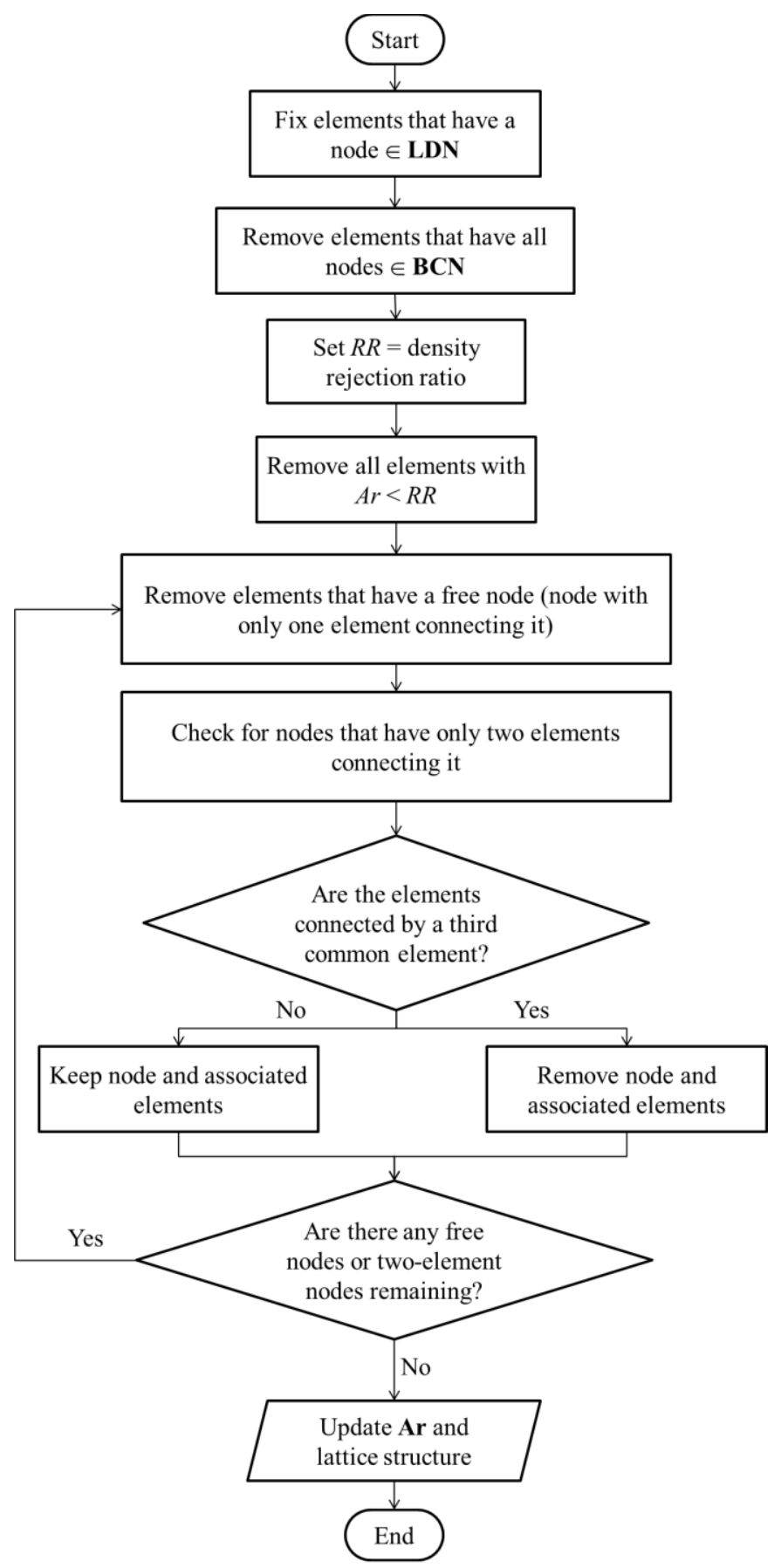

Figure 5 Flowchart of the screening process

trivial if such struts were left. Removing these struts would allow more material to be distributed to the important struts that remain. Figure 6 shows an example of this process. In Figure 6 (a), after the removal of the struts with $A r<R R$, there are struts that remain that are unimportant to the structure. The process will examine all free nodes in the structure, shown in the example, and will find only one node that will be removed. After removing the free node, nodes that connect two struts which share a common third strut are removed. This process continues on till no nodes that meet the criteria remain. Figure 6 (b) shows the final structure after the removal process. 


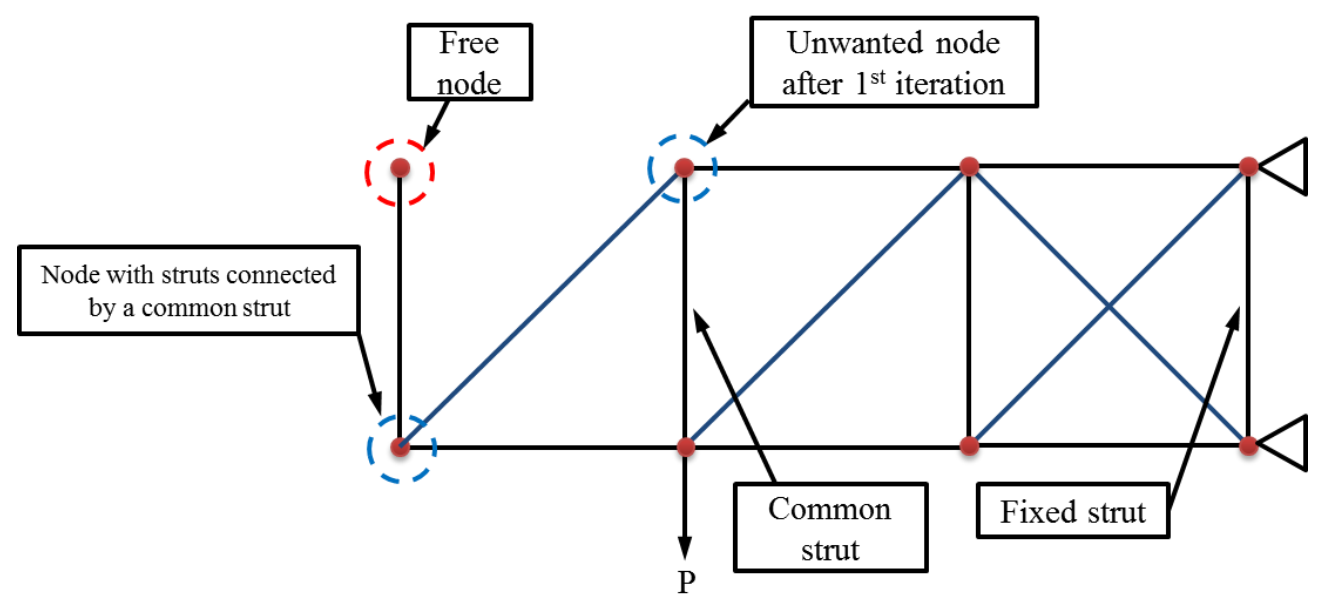

(a)

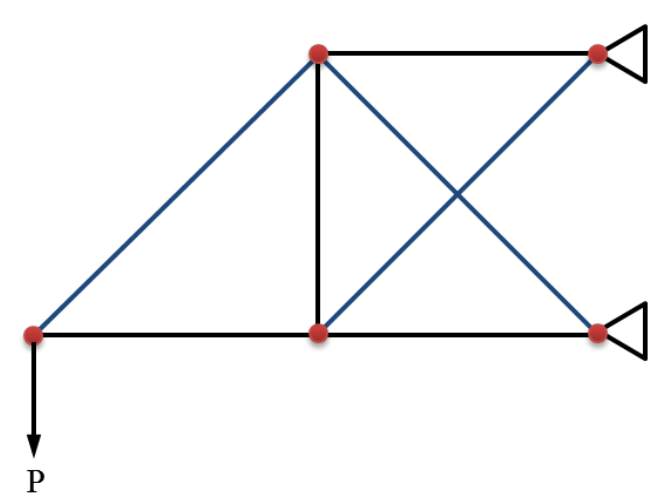

(b)

Figure 6 Illustration of the proposed screening process: a) Initial structure before the screening process b) Final structure after the screening process

\section{Demonstration Examples}

\subsection{2-D Cantilever Beam}

This example demonstrates the application of the RDM method in generating a 2-D lattice structure for a cantilevered beam. It also shows the effects of different element sizes and different $A r_{l}$ and $R R$ values on the performance of the generated structure. The example is similar to the one in Ref. [23] which is of a cantilevered beam loaded in the middle of the tip as shown in Figure 7. Figure 7(b) shows the results of the topology optimization of the beam, where the objective was to reduce the strain energy under a $20 \%$ volume fraction constraint. 1600 linear plane stress elements were used in the optimization. The properties of the beam are summarized in Table 1. A penalization factor of 3 was used in the optimization process in all examples in this paper. The use of the penalization factor is to demonstrate the applicability of the proposed method to already-obtained topology optimization results, and that no special consideration must be taken while conducting the optimization process in order to use the RDM method.

The lattice structure was first generated with a base unit-cell size of $5 \times 5 \mathrm{~mm}$ and a total of 74 struts, and then compared with the ground structure approach using LM and Active-set 
algorithms. An identically sized lattice structure was also created to act as an upper bound to the performance of the generated lattice structures. The objective function for the ground structure approach was formulated as follows

$$
\operatorname{minZ}(\mathbf{D})=\sum_{j=1}^{N}\left[\left(w_{d} \times \delta\left(D_{j}\right)\right)^{2}+\left(w_{v} \times \frac{V\left(D_{j}\right)-V_{0}}{V_{0}}\right)^{2}\right]
$$

where $w_{d}$ and $w_{v}$ are weight variables of values of 1 and 100 for the displacement and volume respectively, $\delta$ is the displacement of the tip and $V$ is the volume of the structure. Both $\delta$ and $V$ are functions of the individual struts' diameters $\mathbf{D}$.

a)

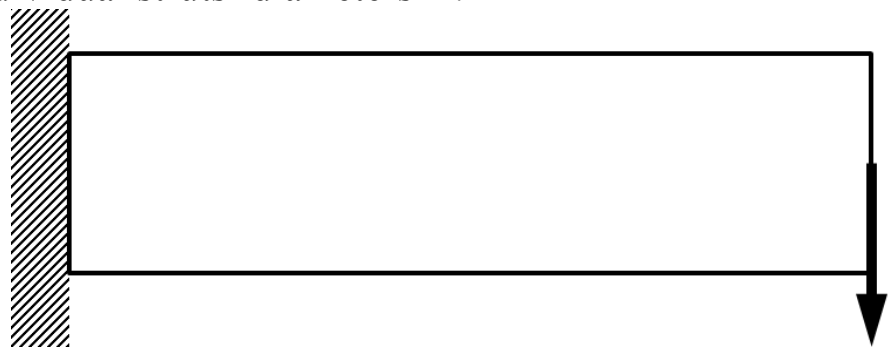

b)

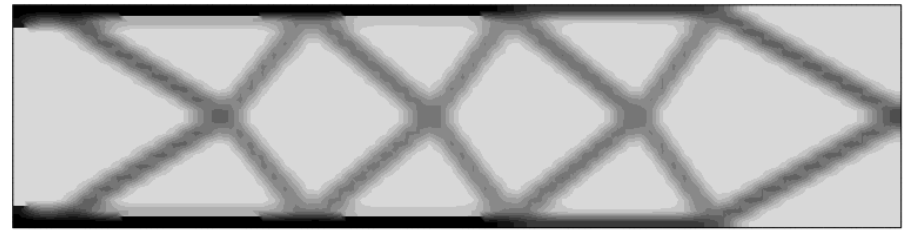

Figure 7 Cantilever beam example: a) Loading condition, b) Topology optimum result

Table 1 Cantilevered beam properties

\begin{tabular}{|c|c|}
\hline Length (mm) & 40 \\
\hline Height (mm) & 10 \\
\hline Thickness (mm) & 1 \\
\hline Loading magnitude (N) & 1 \\
\hline Modulus of Elasticity (MPa) & 1960 \\
\hline Unit-cell size in-plane (mm) & 5 \\
\hline Unit-cell size out-plane (mm) & 5 \\
\hline Target volume (mm3) & 80 \\
\hline
\end{tabular}

Figure 8 shows the generated base lattice structure and the obtained design using RDM with $A r_{l}=0.001$ and $R R=0$ (no screening process). The results of the analysis are shown in Table 2 . The ground structure approach produced the smallest tip displacement of $0.1447 \mathrm{~mm}$ when compared to the results from RDM and the identically sized structure. The RDM method result had a displacement of $0.464 \mathrm{~mm}$, which is relatively greater than the ground structure but $42 \%$ lower than the identically sized structure's result. The solution times for the ground structure approach using Active-Set and LM algorithm were 514.57 and 239.91s respectively. The 
solution times for the RDM method were 1,309 s to conduct the topology optimization process and only $0.13 \mathrm{~s}$ to generate the lattice structure from the information obtained from topology optimization. The RDM method required more time because of the small number of design variables to consider. The next examples will show how the increase in the number of design variables would be advantageous for the RDM method relative to the ground structure approach. If the relative density information of the topology optimization was already available, the RDM method would have a drastic reduction in design time compared to the ground structure approach.
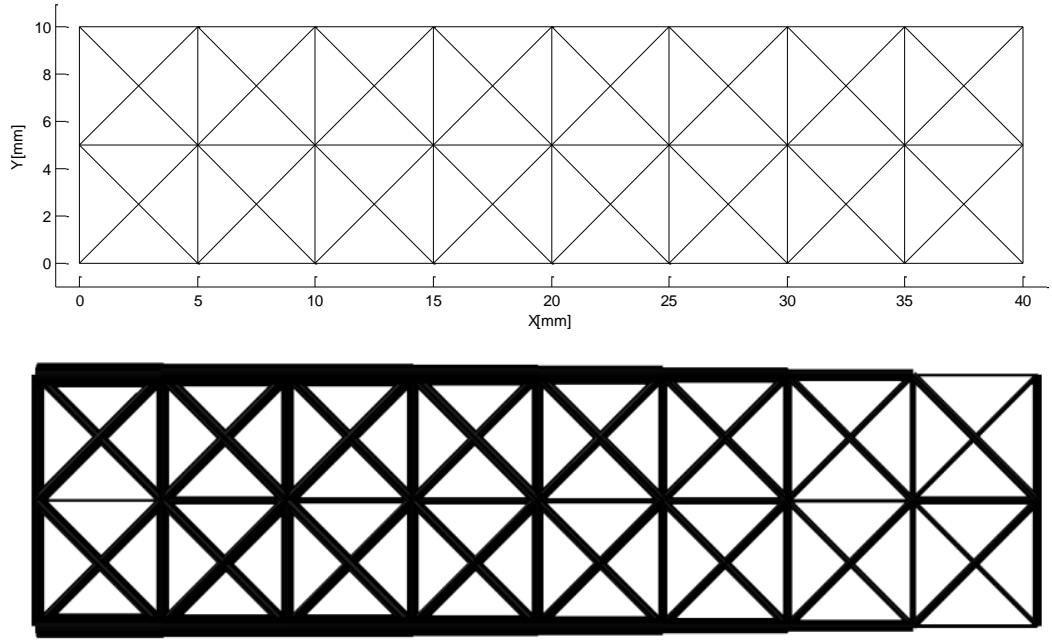

Figure 8 Base lattice structure and generated structure using RDM

Table 2 Optimization results for 2-D beam

\begin{tabular}{|c|c|c|c|}
\hline Method & Tip displacement $(\mathrm{mm})$ & \multicolumn{2}{|c|}{ Solution time (s) } \\
\hline Identically sized structure & 1.14 & \multicolumn{2}{|c|}{-} \\
\hline Ground structure (Active-set) & 0.1447 & \multicolumn{2}{|c|}{514.57} \\
\hline Ground structure (LM) & 0.3657 & \multicolumn{2}{|c|}{239.91} \\
\hline RDM (Arl = 0.001) & 0.464 & 1309 & 0.13 \\
\hline
\end{tabular}

To address the effect of the base unit-cell size and different $A r_{l}$ and $R R$ values, three structures are generated with the RDM method using the same topology optimization results. The three different sizes of the element were $2.5 \times 2.5 \mathrm{~mm}, 5 \times 5 \mathrm{~mm}$ and $5 \times 10 \mathrm{~mm}$. The selected $A r_{l}$ are $0.001,0.01,0.05,0.1,0.15$ and 0.2 while the values of $R R$ are changed from 0.001 to 0.2. The effects of these parameters on the tip displacement are shown in Figure 9. As shown in the figure, an increase in $R R$ value will lead to a reduction in the tip displacement until a certain value where a sudden increase appears in the displacement. The sudden increase in the tip displacement is caused by the removal of important struts in the structure which were essential for the structure's integrity. This can be mitigated by changing $A r_{l}$ values. In most cases, increasing $A r_{l}$ can help in reducing the effects of large $R R$ values, since the important struts will have higher relative densities. The stepped shape of the curves in the figures is due to the structure being unaffected by small changes in $R R$ values. This is clearly noticeable in structures with large base unit-cell sizes. The $5 \times 10 \mathrm{~mm}$ structure had the least tip displacement from all of three structures at values of $A r_{l}=0.1$ and $R R=0.015$. Since all models used the same element size in the topology optimization results, the structure with smaller base 
unit-cell size had fewer elements that had an effective contribution in the determination of the strut's size. This shows the importance of the base unit-cell size with respect to the elements' size used in topology optimization. Another reason is due to the conformity of the crossed lattice struts to the topology optimization results as shown in Figure 10. The $5 \times 10 \mathrm{~mm}$ structure had most of its struts falling in regions with high relative density elements.

a)

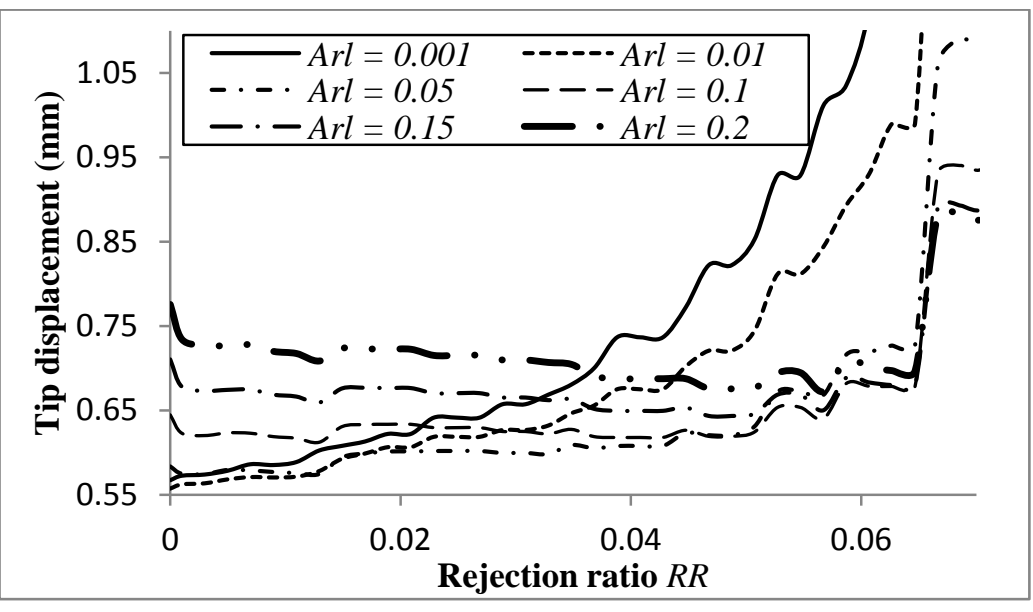

b)

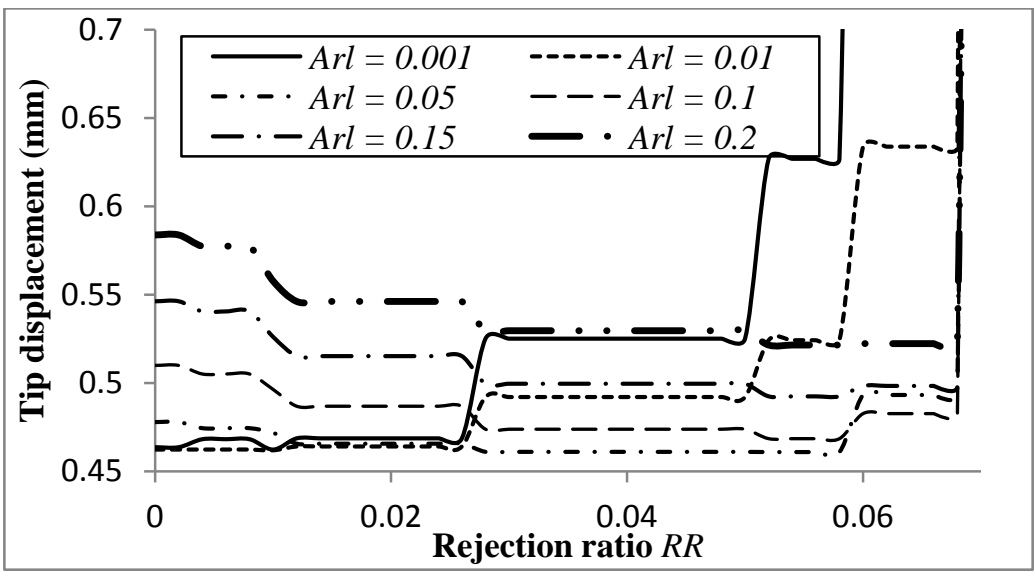

c)

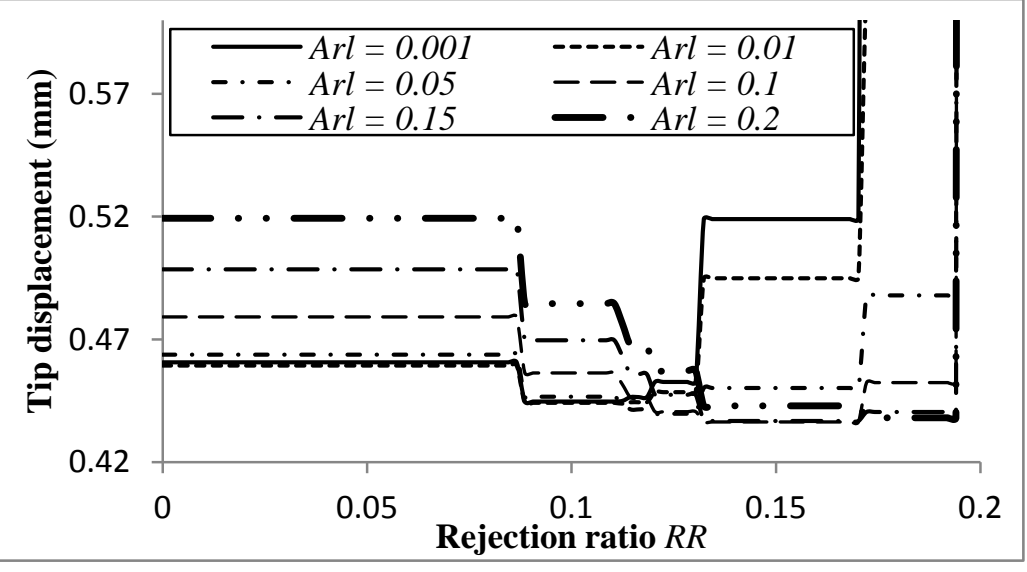

Figure 9 Effect of the values of $\boldsymbol{R} \boldsymbol{R}$ on the tip displacement at different $\boldsymbol{A} \boldsymbol{r}_{\boldsymbol{l}}$ values $2.5 \times 2.5 \mathrm{~mm} \mathrm{~b}) 5 \times 5 \mathrm{~mm} \mathrm{c)} 5 \times 10 \mathrm{~mm}$ 
Based on these observations, a relatively small value of $A_{r l}$ is recommended in the proposed RDM method. A small value of $R R$ is also recommended with small values of $\mathrm{A} r_{l}$, since that will reduce the risk of removing important elements. For the next example, the screening process will be omitted $(R R=0)$ and a small value of $A r_{l}$ will be chosen $\left(A r_{l}=0.01\right)$, since smaller values show no significant improvement in the structure.

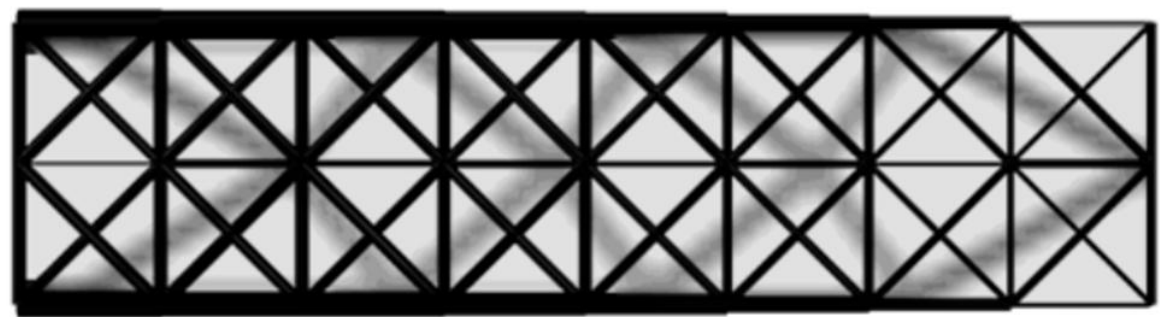

a)

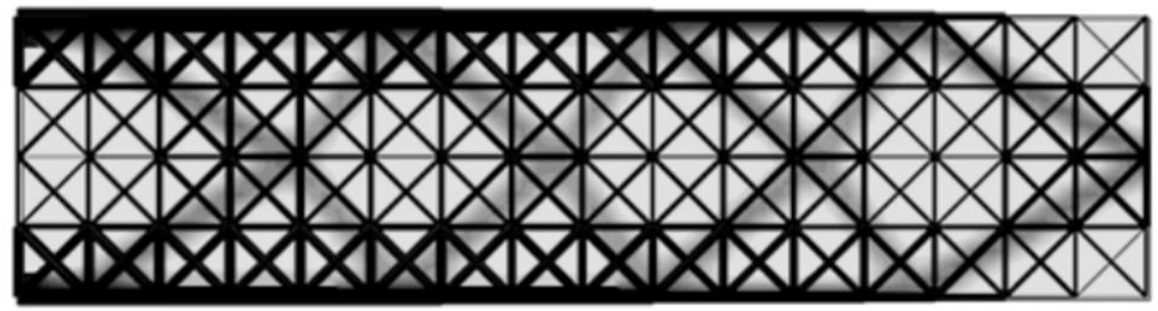

b)

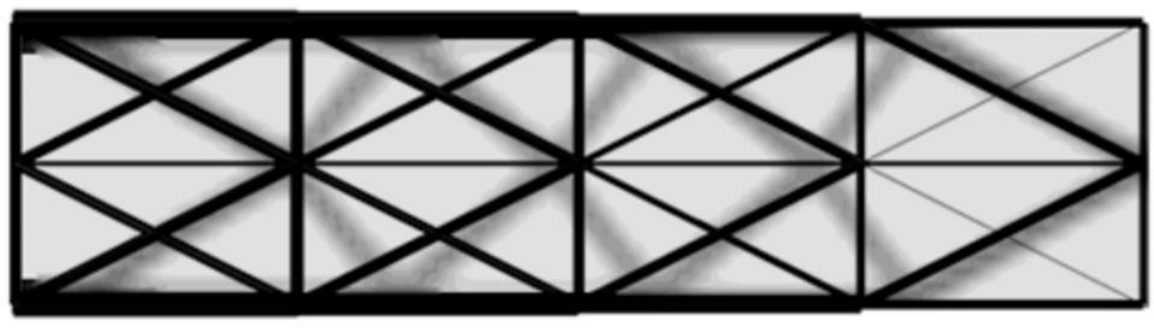

Figure $10 \mathrm{RDM}$ mapping on the topology optimization results: a) $5 \times 5 \mathrm{~mm}$ b) $2.5 \times 2.5 \mathrm{~mm} \mathrm{c)} 5 \times 10 \mathrm{~mm}$

\subsection{Two Load Case 3-D Cantilever Beam}

The second example will show the performance of the RDM method in handling two load cases. The method will be compared to the results obtained by using the ground structure approach and the SMS method with Active-set and LM algorithms. Figure 11 shows the 3-D beam subject to two loading conditions, and Table 3 shows the properties of the beam used. The beam has $50 \mathrm{~mm}$ length, $20 \mathrm{~mm}$ height and the thickness of $10 \mathrm{~mm}$. At the tip of the beam, two point loads of $10 \mathrm{~N}$ acting in the $Y$-direction in one case, and in the $Z$-direction in the other were applied. In order to obtain the density information from the topology optimization, the following optimization statement is considered,

$$
\begin{array}{ll}
\min & U_{1}(\boldsymbol{\rho})+U_{2}(\boldsymbol{\rho}) \\
\text { Subject to } & \sum_{\mathrm{i}=1}^{\mathrm{n}} \rho_{\mathrm{i}} V_{i} \leq 1600 \mathrm{~mm}^{3} \\
& 0 \leq \rho \leq 1
\end{array}
$$

where $U_{1}$ and $U_{2}$ are the strain energies for load case 1 and 2 respectively. A total of 10,000 linear hexahedral elements with 11,781 nodes were used in the process. Figure 12 shows the 
results of topology optimization and the isosurface contour plot of the beam. The topology optimization results show that the unloaded node region would have elements with low relative density values. The isosurface extracted from the optimization process assumes that all elements with relative density of 0.3 or higher to be a solid, and any elements less than that to be voids. Using higher values for the relative density would cause the structure to have discontinuities that would weaken the structure, since elements that are considered essential for the structure's integrity would be removed. This causes the issue of exceeding the volumetric constraint. The extracted geometry had a volume of $2,095 \mathrm{~mm}^{3}$ and requires shape optimization before fabrication. This is where the RDM method would be used, since the generated structures do not need to undergo additional shape optimization procedures while still satisfying the volume constraint. Further discussion on this will be presented in the next example.

RDM was used to generate the lattice structure as shown in Figure 13. The structure had 135 struts, which is higher than the number in the previous example. The generated structure had struts with small diameter values near the zone of the unloaded node in the topology optimization, as expected. This result is then compared with other methods including SMS as shown in Table 4. Since SMS is not able to handle multiple load cases, three different designs are generated with SMS. Two of the structures are generated from each load case separately, and the third is generated by combining both load cases.

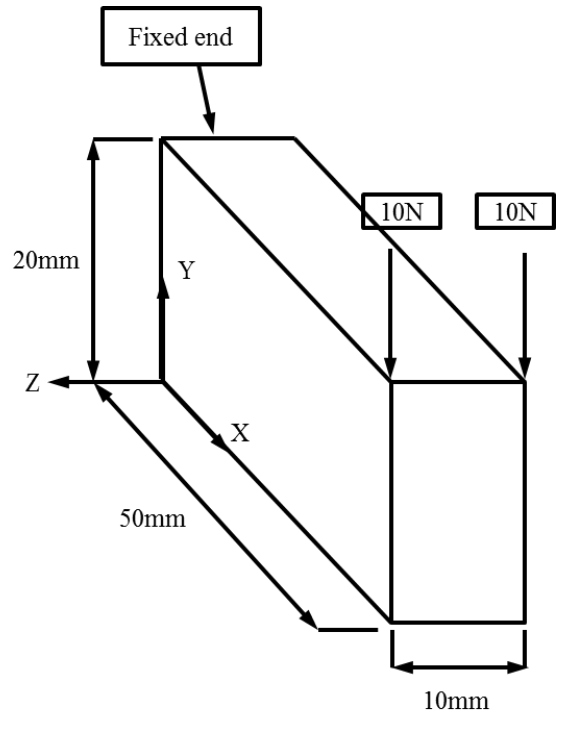

a)

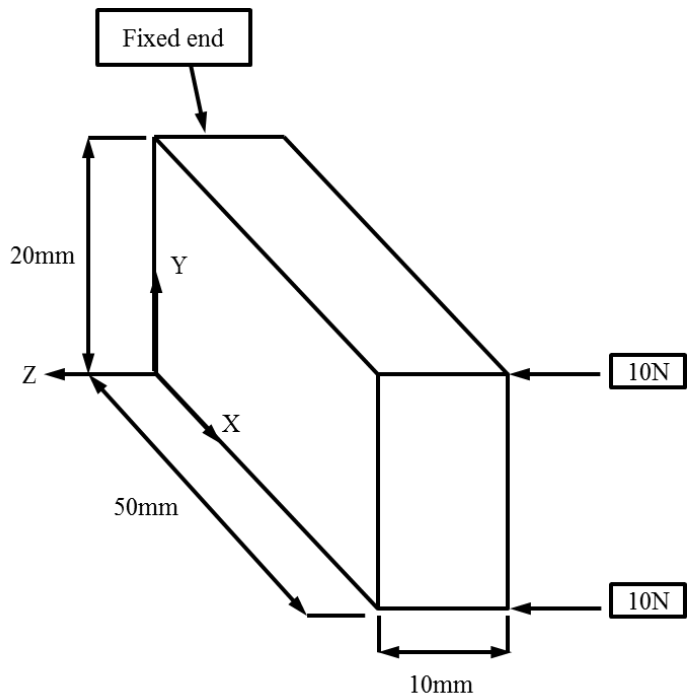

b)

Figure 11 Boundary and Loading condition of Cantilever beam: a) Load case 1 b) Load case 2

Table 3 Cantilever beam properties 


\begin{tabular}{|c|c|}
\hline Length $(\mathrm{mm})$ & 50 \\
\hline Height $(\mathrm{mm})$ & 20 \\
\hline Width $(\mathrm{mm})$ & 10 \\
\hline Loading magnitude $(\mathrm{N})$ & 10 \\
\hline Modulus of Elasticity $(\mathrm{MPa})$ & 1960 \\
\hline Unit-cell size $(\mathrm{mm} \times \mathrm{mm})$ & $10 \times 10$ \\
\hline Target volume $\left(\mathrm{mm}^{3}\right)$ & 1600 \\
\hline
\end{tabular}

The results of the analysis show that the ground structure approach has the least displacement for all structures in both load cases, but requires 4,297.81s to complete the optimization process. The SMS results have the worst performances when compared to the other methods. Considering only the first load case, SMS with active-set and LM algorithms exhibit $Y$-directional displacements of $0.6234 \mathrm{~mm}$ and $0.6225 \mathrm{~mm}$, respectively. However, the displacement in the $Z$-direction is very large when compared to the case of the identically sized structure. This observation is expected since the SMS method does not consider multiple loading conditions. By using the second load case, it is expected to have a reversed effect on the tip displacement. However, the results show higher values in the $Z$-direction. This is caused by the large size of the base unit-cell in the thickness direction of the beam that was used in the solid body FEA, unlike in the $Y$-direction where two unit-cells were used. Using both load cases results in an improvement in the $Y$-direction, but worse results for the displacement in the Z-direction. Unfortunately, the results are still higher than the identically sized structure. However, the SMS method had the shortest time compared to the other methods. The RDM method took 2,541s for the topology optimization, which is $40 \%$ lower than the time required by the ground structure approach due to the increase in the number of design variables. If the relative density information was present, it would have taken $0.76 \mathrm{~s}$ to generate the lattice structure. The displacement obtained from RDM is larger than that of the ground structure in the $Z$-direction, but much lower than the results from the identically sized structure. This shows the benefit of RDM in designing lattice structures that are subject to multiple loading conditions.

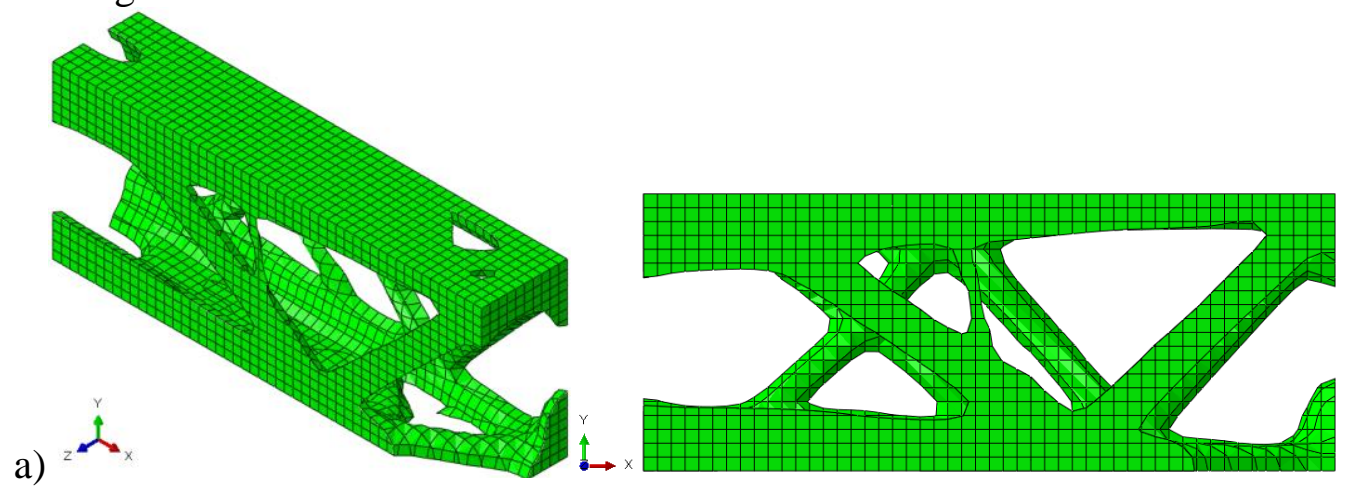


b)
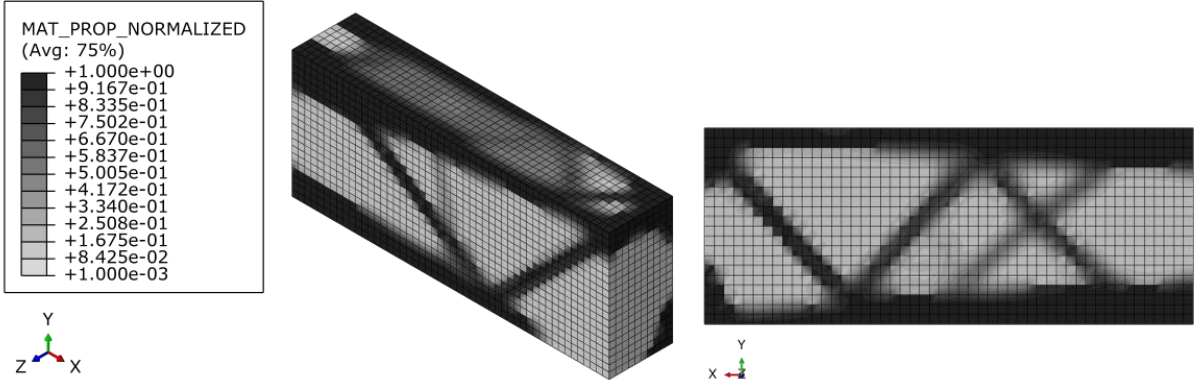

Figure 12 Topology optimization results: a) Isosurface rendered from the topology optimization and b) Relative density contour plot

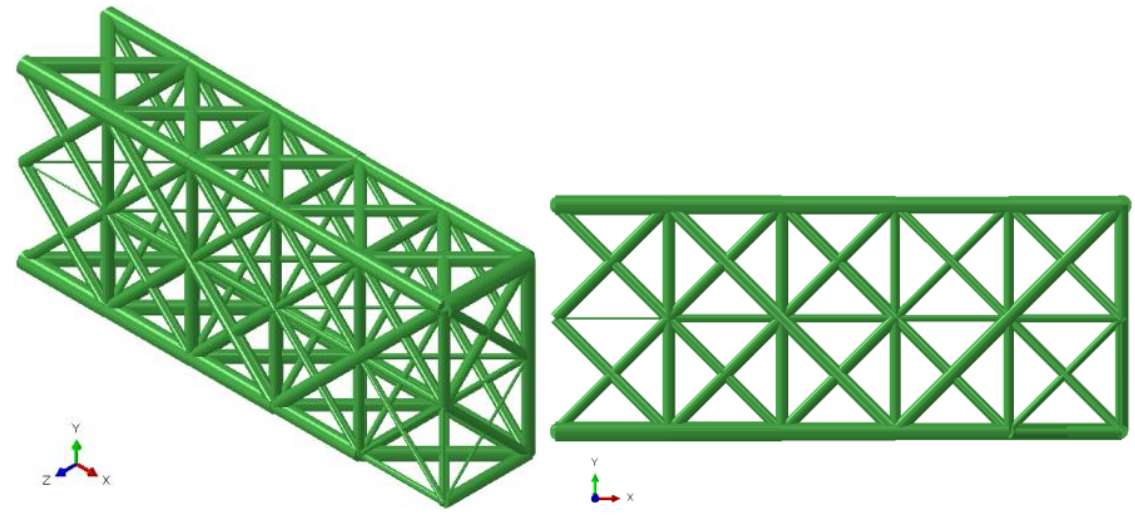

Figure 13 Generated lattice structure using RDM

Table 4 Results of the optimization methods of the cantilever beam

\begin{tabular}{|c|c|c|c|c|c|}
\hline & Method & $\begin{array}{c}\text { Displacement } \\
\text { Y } \\
(\mathrm{mm})\end{array}$ & $\begin{array}{c}\text { Displacement } \\
\text { Z } \\
(\mathrm{mm})\end{array}$ & \multicolumn{2}{|c|}{ Solution time (s) } \\
\hline & tically sized structure & 1.21 & 2.837 & \multicolumn{2}{|c|}{-} \\
\hline & d structure (Active-set) & 0.5523 & 0.996 & \multicolumn{2}{|c|}{$4,297.81$} \\
\hline$\overleftarrow{\circlearrowright}$ & SMS (Load case 1) & 0.6234 & 29.1 & \multicolumn{2}{|c|}{21.10} \\
\hline$\stackrel{1}{2}$ & SMS (Load case 2) & 3.668 & 6.2 & \multicolumn{2}{|c|}{9.01} \\
\hline & SMS (combined Loads) & 3.024 & 7.626 & \multicolumn{2}{|c|}{9.79} \\
\hline & SMS (Load case 1) & 0.6225 & 29.3 & \multicolumn{2}{|c|}{19.76} \\
\hline$\sum_{\Delta}$ & SMS (Load case 2) & 3.668 & 6.2 & \multicolumn{2}{|c|}{22.72} \\
\hline & SMS (combined Loads) & 3.024 & 7.626 & \multicolumn{2}{|c|}{25.69} \\
\hline & $\operatorname{RDM}\left(A r_{l}=0.01\right)$ & 0.723 & 1.83 & 2,541 & 0.759 \\
\hline
\end{tabular}

\subsection{Micro Aerial Vehicle Fuselage}

In this section, a fuselage design example of a Micro Aerial Vehicle is considered [1]. MAVs are used for surveillance in hazardous areas, which makes them critical in military operations. They are designed to have high performance in endurance, landing and take-off [29]. Light weight and durable materials are desired for designing the fuselage and wings of 
these MAVs. The fuselage is designed so that it can handle impact loads from landing or crashing.

The example will demonstrate the applicability of the RDM method in structures with curved and non-rectangular surfaces. Figure 14 shows two views of the side section of the fuselage with key dimensions labeled. Table 5 shows the properties and design parameters of the fuselage and the unit-cell used in the example. Figure 15 shows the loading and boundary conditions on the fuselage. There are three loads on the fuselage: the weight of the motor, the weight of the tail, and a payload that acts as a distributed load on the inner surface of the fuselage. The wings of the MAV have such a small weight that their effect was neglected. To model the impact loading on the fuselage, all loads are scaled by a factor of ten (Table 5 values are scaled). The contact area between the MAV and the ground is assumed to be fixed to model the impact zone.
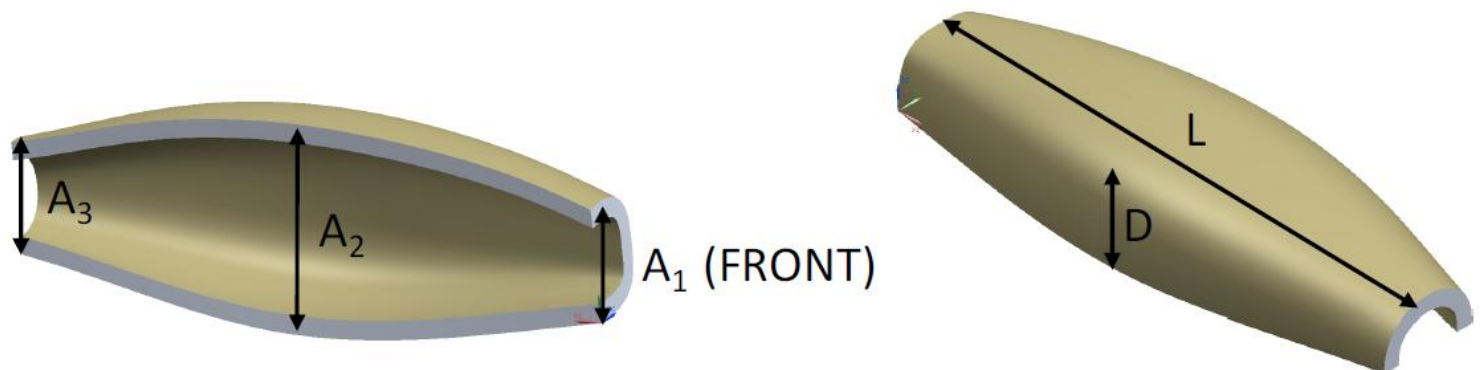

Figure 14 General dimension labels of the fuselage [1]

Table 5 intial properties for the fuselage

\begin{tabular}{lclc}
\hline $\mathrm{A}_{1}(\mathrm{~mm})$ & 47 & $\mathrm{~L}(\mathrm{~mm})$ & 254 \\
\hline $\mathrm{A}_{2}(\mathrm{~mm})$ & 90 & $\mathrm{~F}_{\text {motor }}(\mathrm{N})$ & 5.9 \\
\hline $\mathrm{A}_{3}(\mathrm{~mm})$ & 45 & $\mathrm{~F}_{\text {Tail }}(\mathrm{N})$ & 2.7 \\
\hline $\mathrm{D}(\mathrm{mm})$ & 45 & $\mathrm{~F}_{\text {Payload }}\left(\mathrm{N} / \mathrm{mm}^{2}\right)$ & 0.1 \\
\hline Unit-Cell size in-plane $(\mathrm{mm})$ & 12 & Modulus $\left(\mathrm{N} / \mathrm{mm}^{2}\right)$ & 1960 \\
\hline Unit-cell size in-plane $(\mathrm{mm})$ & 12 & Poisson Ratio & 0.3 \\
\hline Unit-cell size out-plane $(\mathrm{mm})$ & 8 & Target Volume $\left(\mathrm{N} / \mathrm{mm}^{3}\right)$ & 100,000 \\
\hline
\end{tabular}

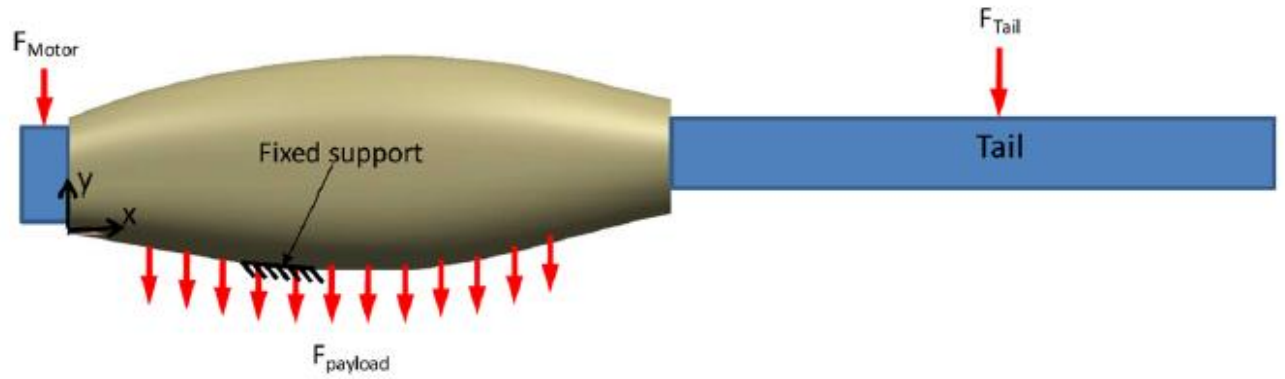

Figure 15 Loading and boundary conditions [1]

To use the RDM method, a continuum topology optimization was carried out to obtain the relative density information with the following statement, 


$$
\begin{array}{ll}
\min & U_{1}(\boldsymbol{\rho}) \\
\text { Subject to } & \sum_{\mathrm{i}=1}^{\mathrm{n}} \rho_{\mathrm{i}} V_{i} \leq 100,000 \mathrm{~mm}^{3} \\
& 0 \leq \rho \leq 1
\end{array}
$$

9,000 linear hexahedral elements with 11,831 nodes were used in the process. The isosurface obtained from the optimization process is shown in Figure 16 along with the contour plot of the relative density of the elements. Figure 17 shows an STL model of the isosurface rendered from the optimization process. It was ensured that the model satisfied the volumetric constraint imposed by the optimization formulation. From the figure, it can be noticed that the model is not suitable to be used for FEM. Each facet on the surface is assumed to be a separate face of the geometry. The ribs on the side of the model also have a very small thickness that would cause further complications. Finer elements would have to be used in order to avoid any numerical errors from poorly shaped elements. Furthermore, shape optimization would be required and would cost more time with fine elements. Using the RDM method, the information of the relative density can be mapped on a predefined structure that would eliminate the need for shape optimization and the cost of the time associated with the process.
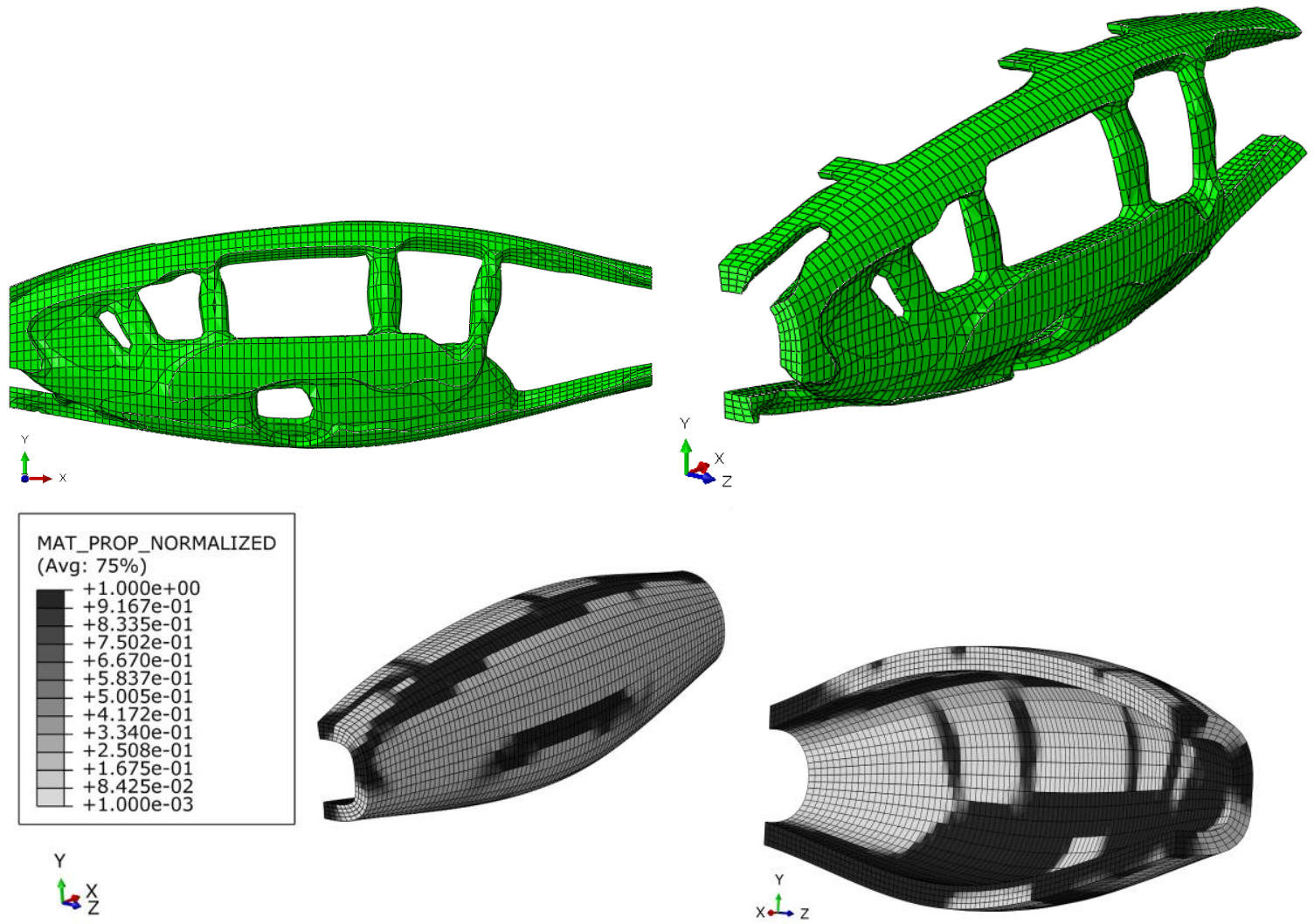

Figure 16 Topology optimization results: a) Isosurface rendered from the topology optimization and b) Relative density contour plot 

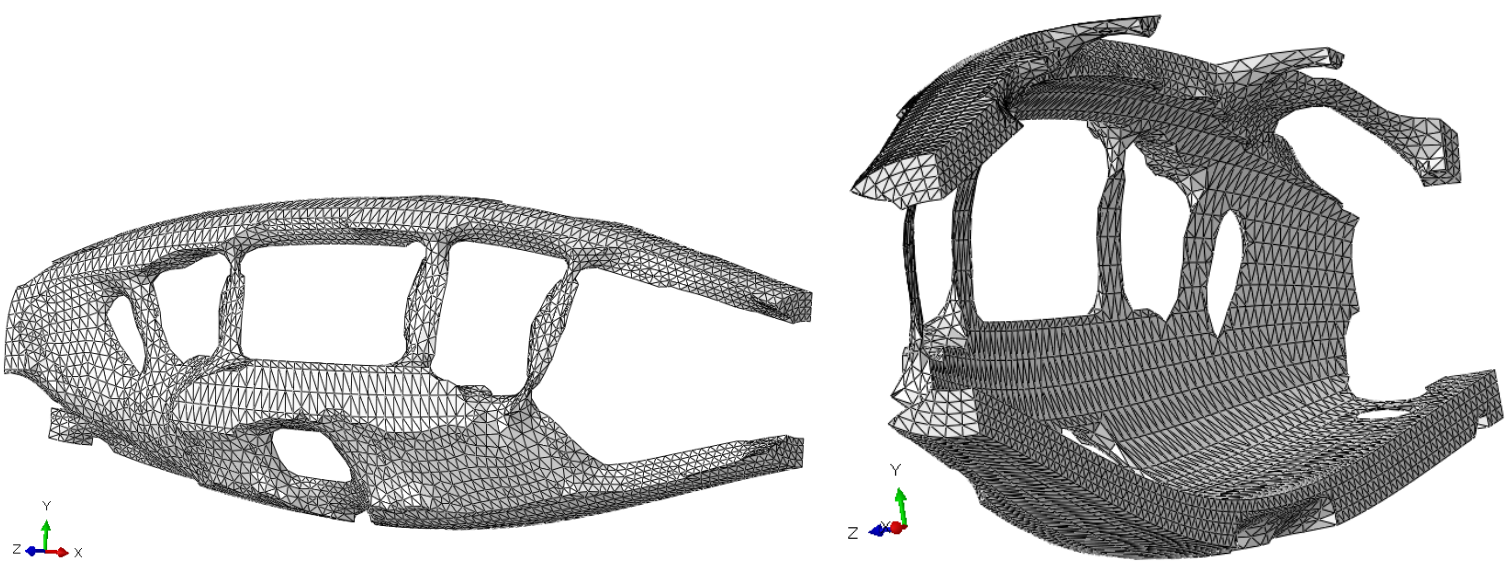

Figure 17 Extracted STL file from the optimizaion process

Figure 18 shows the lattice structure that was generated using these relative densities, which had 2081 struts. This is much higher than the previous examples. The performance of the obtained structure is compared to the results found in Ref. [1], which are shown in Table 6.
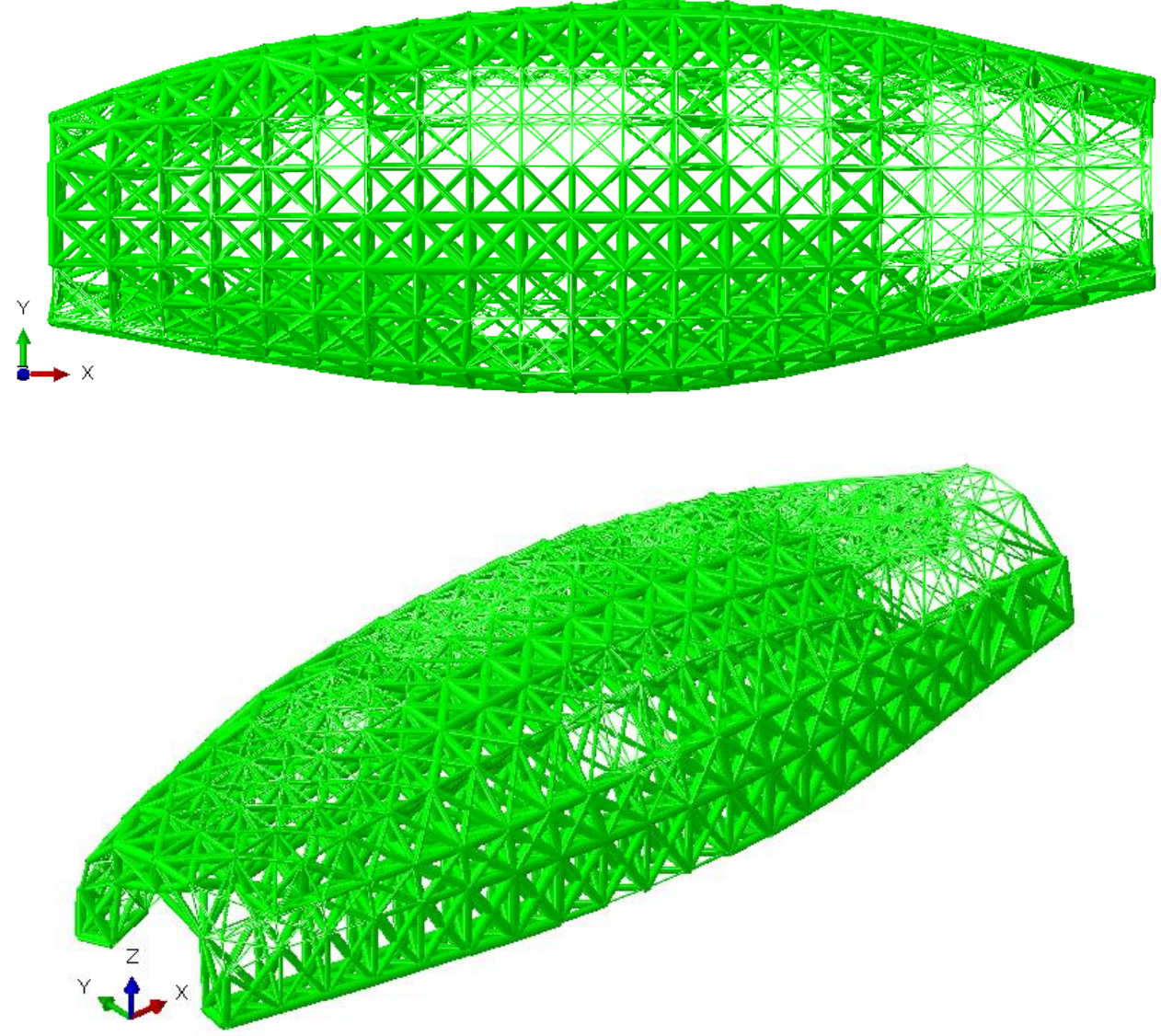

Figure 18 Generated lattice structure using RDM 
Table 6 Results of the optimization methods for MAV

\begin{tabular}{|c|c|c|c|}
\hline Optimization method & Deflection $(\mathrm{mm})$ & \multicolumn{2}{|c|}{ Time $(\mathrm{s})$} \\
\hline Identically sized & 0.425 & \multicolumn{2}{|c|}{-} \\
\hline SMS (Active-Set) & 0.299 & \multicolumn{2}{|c|}{$1,630.9$} \\
\hline SMS (LM) & 0.319 & \multicolumn{2}{|c|}{508.9} \\
\hline RDM & 0.3431 & 1,900 & 3.64 \\
\hline
\end{tabular}

RDM took more time than SMS in generating the structure, but much less time than the ground structure approach. The results from RDM are comparable to SMS with least square minimization algorithm, and below the results of the identically sized structure. The ground structure approach was not used due to convergence issues with the sheer number of design variables to consider. The time to run the ground structure approach would be much longer than the RDM methods. As the second example showed, SMS would not be able to generate an optimized structure under multiple loading conditions. So if the structure was to be designed to handle more than one loading, SMS would perform poorly. This would be advantageous to the RDM method, since the ground structure wouldn't be a feasible choice in this case.

\section{Conclusion}

A new design method for truss-like cellular structures is developed. The proposed method utilizes the by-product information obtained from topology optimization for the first time. This method does not require additional computational costs for conducting the optimization or FEM simulations. The proposed method was compared with the SMS method and the ground structure approach to investigate its performance on a cantilevered beam subject to two loading conditions. Another comparison was conducted between the RDM and SMS method on the design of the fuselage of a MAV. Based on the comparison results, several aspects are observed:

- The selection of $R R$ and $A r_{l}$ values can help in improving the performance of the structure. The selection of a reasonable $R R$ value should be based on the size of the unit cells with respect to the part size. Large unit cells would require large $R R$ values, since $A r$ for each strut would be significantly large and different from strut to strut. But parts with small unit cells will tend to have struts that have close values of $A r$, which would require small RR values.

- The results of the second example showed that RDM has better performance than SMS when handling multiple loading conditions. SMS also performed poorly due to the large size of unit-cells relative to the solid body since only one unit-cell was used in the $Z$-direction.

- When compared to the ground structure approach, RDM had higher displacement than the ground structure approach, but the ground structure approach required significantly more time as the number of design variables increased.

\section{Acknowledgement}

This work was partially supported by the National Science Foundation under Grant Number CMMI-1200788. 


\section{References}

[1] J. Nguyen, S. Park and D. W. Rosen, "Heuristic optimization method for cellular structure design of light weight components," International Journal of Precision Engineering and Manufacturing, vol. 14(6), pp. 1-8, 2013.

[2] M. R. Legault and M. Musselman, "The rise of rapid manufacturing," High Performance Composites, vol. 17(4), pp. 32-37, July 2009.

[3] D. L. Bourell, J. B. Beaman, M. C. Leu and D. W. Rosen, "A brief history of additive manufacturing and the 2009 roadmap for additive manufacturing: looking back and looking ahead," In US-Turkey Workshop on Rapid Technologies, vol. pp. 2009.

[4] D. W. Rosen, "Computer-aided design for additive manufacturing of cellular structures," Computer-Aided Design \& Applications, vol. 4(5), pp. 585-594, 2007.

[5] D. W. Rosen and I. Gibson, Additive Manufacturing Technologies: Rapid Prototyping to Direct Digital Manufacturing. Springer, 2009.

[6] L. J. Gibson and M. F. Ashby, Cellular Solids: Structure and properties. Cambridge: Cambridge University Press, 1997.

[7] M. F. Ashby and R. F. Mehl Medalist, "The mechanical properties of cellular solids," Metallurgical Transactions A, vol. 14(9), pp. 1755-1769, 1983.

[8] M. F. Ashby, A. G. Evans, N. A. Fleck, L. J. Gibson, J. W. Hutchinson and H. N. G. Wadley, Metals Foams: A Design Guide. Burlington, MA: Butterworth-Heinemann, 2000.

[9] B. Wang, G. Zhang, Q. He, L. Ma, L. Wu and J. Feng, "Mechanical behavior of carbon fiber reinforced polymer composite sandwich panels with 2-D lattice truss cores," Materials and Design, vol. 55(pp. 591-596, 2014.

[10] S. Yin, L. Wu and S. R. Nutt, "Compressive efficiency of stretch-stretch-hybrid hierarchical composite lattice cores," Materials and Design, vol. 56(pp. 731-739, 2014.

[11] V. S. Deshpande, N. A. Fleck and M. F. Ashby, "Effective properties of the octet-truss lattice material," Journal of the Mechanics and Physics of Solids, vol. 49(2001), pp. 1747-1769, 2001.

[12] J. C. Wallach and L. J. Gibson, "Mechanical behavior of a three-dimensional truss material," International Journal of Solids and Structures, vol. 38(pp. 7181-7196, 2000.

[13] S. Chiras, D. R. Mumm, A. G. Evans, N. Wicks, J. W. Hutchinson, K. Dharmasena, H. N. G. Wadley and S. Fichter, "The structural performance of near-optimized truss core panels," International Journal of Solids and Structures, vol. 39(pp. 4093-4115, 2002.

[14] H. V. Wang, C. Williams and D. W. Rosen, "Design Synthesis of Mesoscopic Cellular Structures With Unit Truss Approach and Particle Swarm Optimization Algorithm," in Proceedings of the The Seventeenth Solid Freeform Fabrication Symposium, Austin, Texas, 2006, pp.

[15] A. G. M. Michell, "The limits of economy of material in frame structures," Philosophical Magazine, vol. 6(47), pp. 589-597, 1904.

[16] W. Dorn, R. Gomory and H. Greenberg, "Automatic design of optimal structures," Journal Mechanica, vol. 3(pp. 25-52, 1964.

[17] J. Patel and S. K. Choi, "Classification approach for reliability-based topology optimization using probabilistic neural networks," Structural and Multidisciplinary Optimization, vol. 45(4), pp. 529-543, 2011.

[18] N. Gould, D. Orban and P. Toint, "Numerical methods for large-scale nonlinear optimization," Acta Numerica, vol. 14(pp. 299-361, 2005. 
[19] W. H. Press, S. A. Teukolsky, W. T. Vettering and B. P. Flannery, Numerical Recipes in C. Cambridge, UK: Cambridge University Press, 1992.

[20] G. C. Graf, J. Chu, S. Engelbrecht and D. W. Rosen, "Synthesis methods for lightweight lattice structures," in ASME IDETC/CIE 2009, San Diego, California, 2009, pp.

[21] P. Chang and D. W. Rosen, "An improved size, matching and scaling method for the design of deterministic mesoscale truss structures," in ASME IDETC/CIE 2011, Washington, DC, 2011, pp.

[22] K. Levenberg, "A method for the solution of certain non-linear problems in least squares," The Quarterly of Applied Mathematics, vol. 2(pp. 164-168, 1944.

[23] M. P. Bendsøe and O. Sigmund, Topology Optimization: Theory, Methods and Applications. Berlin: Springer, 2003.

[24] M. P. Bendsøe and N. Kikuchi, "Generating optimal topologies in structural design using a homogenization method," Computer Methods in Applied Mechanics and Engineering, vol. 71(pp. 197-224, 1988.

[25] O. Sigmund, "A 99 line topology optimization code written in Matlab," Structural Multidisciplinary Optimization, vol. 21(2), pp. 120-127, 2001.

[26] G. I. N. Rozvany, M. Zhou and T. Birker, "Generalized shape optimization without homogenization," Structural Optimization, vol. 4(3-4), pp. 250-252, 1992.

[27] O. Sigmund and P. M. Clausen, "Topology optimization using a mixed formulation: An alternative way to solve pressure load problems," Computer Methods in Applied Mechanics and Engineering, vol. 196(13-16), pp. 1874-1889, 2007.

[28] K. Svanberg and M. Stople, "An alternative interpolation scheme for minimum compliance topology optimization," Structural Multidisciplinary Optimization, vol. 22(2), pp. 116-124, 2001.

[29] DARPA Public Affairs, "Can You Design, Build, and Fly the Next Generation UAV?" http://science.dodlive.mil (Accessed April 1 2015) 
Topology optimization

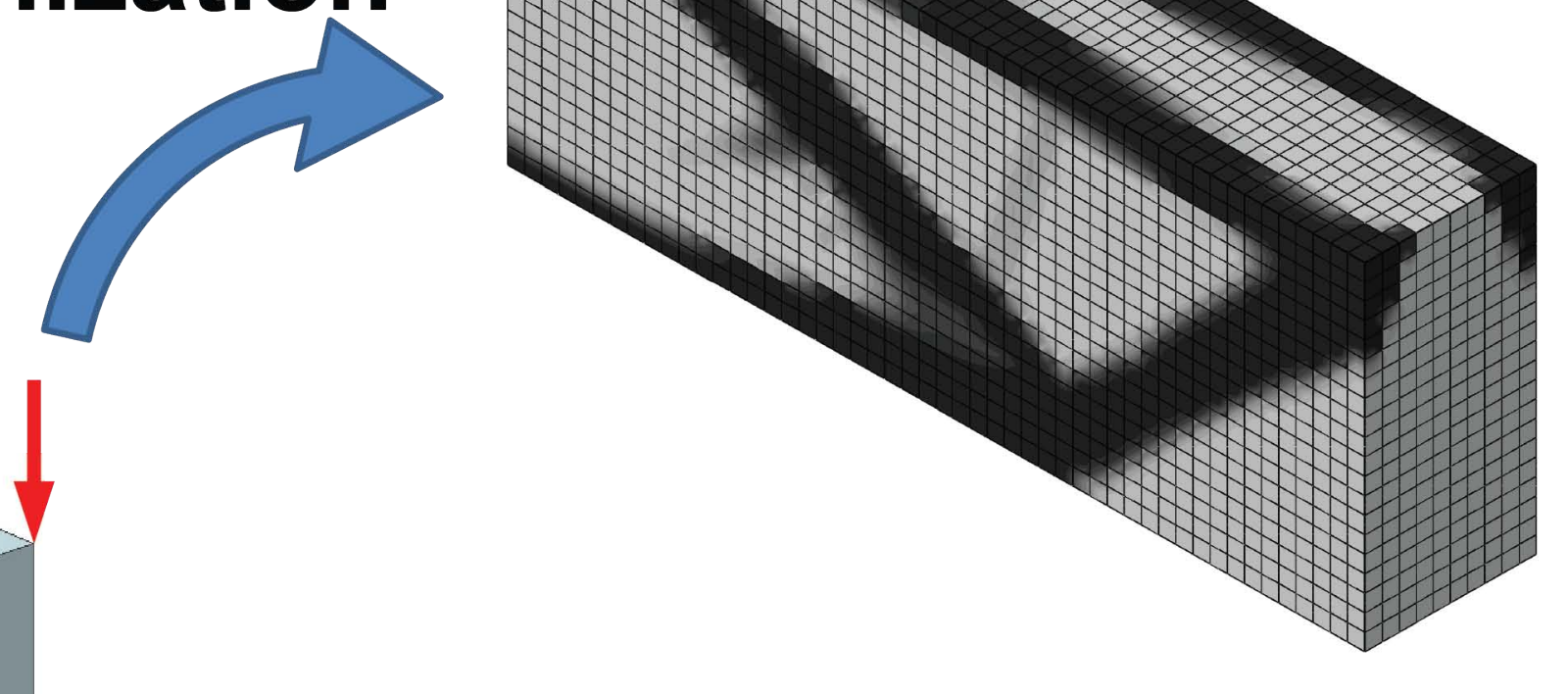

Relative Density Mapping (RDM)

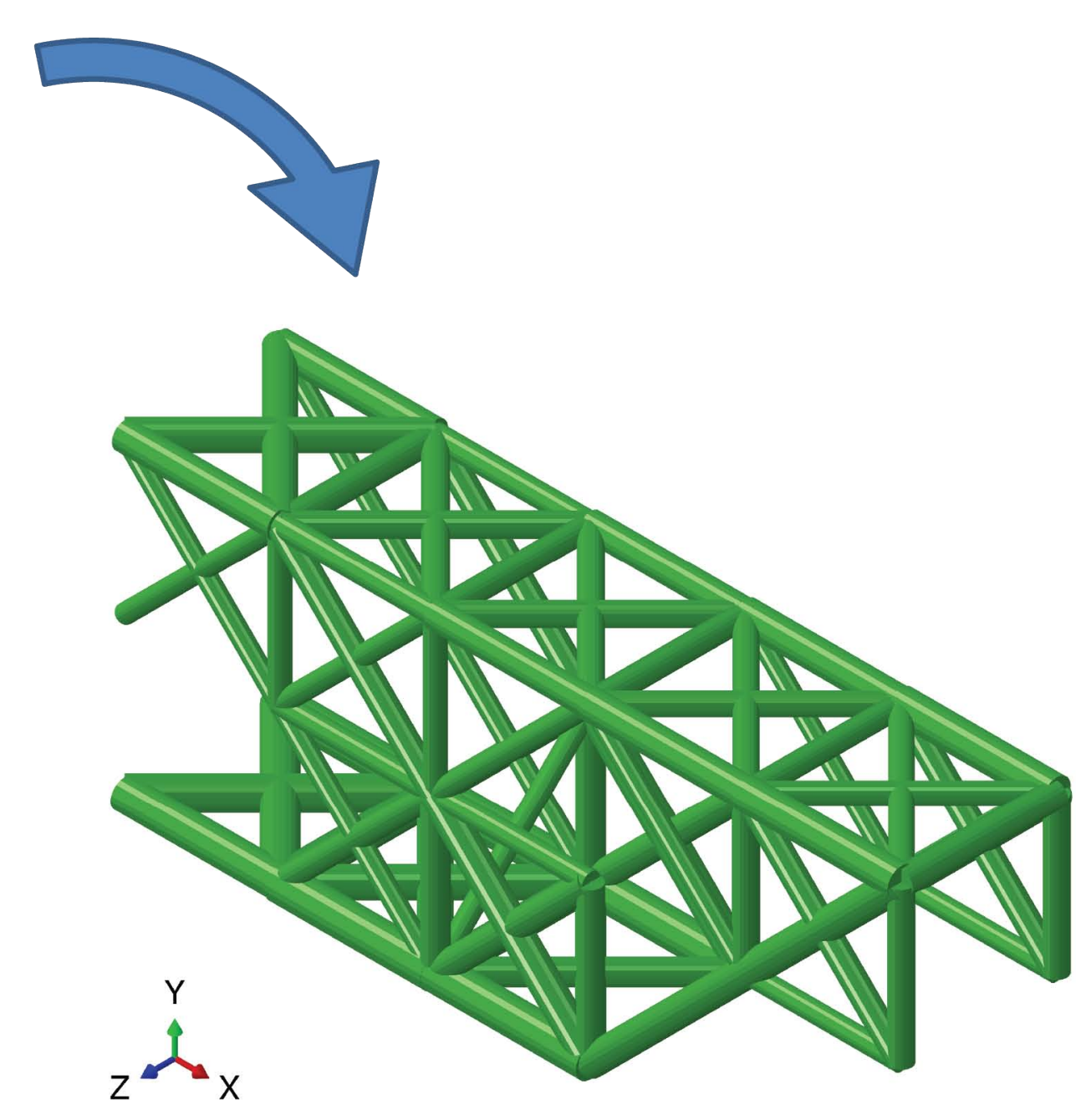

